\title{
PRECISE AND ROBUST MAGNETIC FIELD COMPUTATIONS FOR HIGH-END SMART SENSOR APPLICATIONS
}

\author{
ANDRÉ BUCHAU \\ Institute of Smart Sensors, University of Stuttgart, Germany
}

\begin{abstract}
Highly precise magnetic field computations are essential for the design of magnets and coils of highend smart sensors in the context of nuclear magnetic resonance (NMR) and electron spin resonance (ESR) applications. Here, requirements for numerical magnetic field computations are that the methods are precise and mainly robust in the sense of reliable results. Non-linear magnetic materials, induced eddy currents, and the infinite surrounding air domain must be accurately considered in the numerical model. To this end, a smart combination of the finite element method (FEM) with the boundary element method (BEM) including a powerful post-processing is proposed with a focus on the region of interest (ROI), which contains the studied sample. Separate BEM domains are defined for the infinite surrounding air domain and the ROI. Then, the computational costs are reduced due to the relatively small number of relevant boundary elements of the surface of the ROI and a precise and efficient evaluation of magnetic fields based on a meshfree BEM post-processing in the ROI is possible. As an example, the numerical formulation, the application of the proposed approach, and numerical results are shown for the homogeneous field of a permanent magnet.
\end{abstract}

Keywords: boundary element method, finite element method, smart sensors, magnetic fields, permanent magnets, nuclear magnetic resonance spectroscopy.

\section{INTRODUCTION}

The concept of a smart sensor is to combine a sensor, which measures a physical value, with a low-noise analogue interface circuit and the digital signal processing in a chip [1]. An implementation of such a smart sensor within an application specific integrated circuit (ASIC) is very advantageous, since it enables in-situ measurements with a high signal-tonoise ratio. An example for a high-end smart sensor application is to measure free radicals in blood samples using a mobile electron spin resonance (ESR) spectrometer [2]. A similar design can be applied for nuclear magnetic resonance (NMR) microscopy [3].

Both for ESR and NMR applications, homogeneous magnetic fields in the region of interest (ROI) are required, which directly influence the quality of the measured signal. Hence, the design of permanent magnets with highly homogenous magnetic fields is of great importance for these applications. To this end, a shimming based on accurate numerical field computations including optimization techniques and precise measurements is necessary [4]. Then, manufacturing tolerances can be compensated, too. Concerning numerical field computations, accuracy of the numerical methods is more important than the computational costs. However, a significant criterion for the selection of an appropriate numerical method is that the numerical model is simple and robust. That means, possible inaccuracies caused by the discretized model of the magnet should be minimized.

Typically, the magnet consists of permanent magnets, which are embedded into a yoke with defined geometrical shape of the pole shoes as a result of the shimming process. The yoke is made of magnetic material with a non-linear relation between the magnetic field strength $\boldsymbol{H}$ and the magnetic flux density $\boldsymbol{B}$. Hence, the finite element method (FEM) is well suited for accurate numerical computations of the magnetic field inside the magnet. A threedimensional FEM model of the magnet takes all relevant effects including stray fields into 
account. Furthermore, arbitrary complex numerical models of the magnetic material properties can be included in the design process of the magnet. However, the truncated surrounding air domain affects the magnetic field in the ROI significantly.

A very elegant approach to tackle the problem of the infinite air domain is to couple the FEM with the boundary element method (BEM). Then, the magnetic fields in the air domain are computed using the BEM. Furthermore, a discretization of the ROI is not necessary and the magnetic fields are not interpolated but directly computed from the related boundary integrals with a high accuracy.

In total, a coupled problem must be solved. Modern numerical techniques like iterative solvers for the linear system of equations (LSE), enhanced integration techniques for singular or nearly singular integrals of the BEM, and matrix compression techniques for the dense BEM matrix blocks enable an efficient solution of the problem.

A very important step in numerical computations of magnetic fields for smart sensor applications is the post-processing. The magnetic fields in the ROI must be computed with high accuracy for an evaluation of the homogeneity of the magnetic field. To this end, a meshfree post-processing of the BEM in the ROI is very advantageous, since no discretization and approximation of the fields is necessary. A modular software concept enables a combination of the computed fields for a visualization of the fields both inside the yoke, which are computed using the FEM, and inside the BEM controlled ROI simultaneously.

Here, an accurate and robust approach for the computation of magnetic fields for smart sensor applications is presented. First, a scalar potential formulation is discussed, which is well suited for this kind of field problems for a solution based on the FEM or the BEM. Starting from this overview, a strategy for a coupled application of both methods is shown. The focus is on an optimization of the BEM domains related to efficiency and accuracy. There, compressed matrix blocks of the corresponding linear equation system as well as boundary integrals for a meshfree post-processing are considered. In total, a computational method is presented, which is easy to use for a smart sensor designer and which delivers reliable and accurate numerical results.

\section{FORMULATION}

A mathematical and numerical formulation for the computation of static magnetic fields is required, which enables the determination of magnetic fields in the ROI reliably with a high accuracy. There, non-linear material properties of the yoke, small geometrical details of the pole shoes, and the infinite air domain must be considered. Furthermore, the formulation should be well suited both for the FEM and the BEM.

Here, a magnetic scalar potential formulation was chosen, since only configurations with permanent magnets as field sources are studied. Then, a scalar potential formulation reduces the computational costs in comparison to a more general applicable vector potential formulation significantly without a loss of accuracy.

First, the scalar potential formulation is revisited with respect to the computation of magnetic fields for NMR and ESR applications. Then, numerical models for the non-linear magnetic material properties are discussed. Finally, the basic equations for the applied FEM and BEM are shown to lay the foundation for the proposed robust field computation approach.

\subsection{Scalar magnetic potential}

Since only permanent magnets are used as sources for the magnetic field, a scalar magnetic potential $\psi$ suffices to compute the magnetic field strength $\boldsymbol{H}$ 


$$
\boldsymbol{H}=-\nabla \psi
$$

An important advantage of the scalar magnetic potential is that the computational costs are significantly smaller than for a more general applicable magnetic vector potential $\boldsymbol{A}$. Hence, a much finer discretization can be applied, especially near corners, edges, or at geometrical small structures, which is important in terms of accuracy.

In a domain with magnetic material properties, the magnetic flux density $\boldsymbol{B}$ is obtained directly from $\boldsymbol{H}$ and the permanent magnet's magnetization $\boldsymbol{M}$

$$
\boldsymbol{B}=\mu_{r} \mu_{0} \boldsymbol{H}+\boldsymbol{M},
$$

with relative permeability $\mu_{r}$ and vacuum permeability $\mu_{0}$. Starting from

$$
\nabla \boldsymbol{B}=0,
$$

a partial differential equation for $\psi$ must be solved in each computational domain

$$
\nabla \mu_{r} \nabla \psi=\frac{1}{\mu_{0}} \nabla \boldsymbol{M} .
$$

The magnetic potential is continuous at domain boundaries

$$
\psi_{1}=\psi_{2} .
$$

Tangential component of $\boldsymbol{H}$ is continuous

$$
\boldsymbol{n}_{12} \times\left(\boldsymbol{H}_{2}-\boldsymbol{H}_{1}\right)=\mathbf{0}
$$

and its normal component is discontinuous

$$
\mu_{0} \mu_{r 1} H_{n 1}+M_{n 1}=\mu_{0} \mu_{r 2} H_{n 2}+M_{n 2} .
$$

From eqn (7) it can be seen that the magnetic field strength in high permeable materials is much smaller than in the adjacent air domain. This yields to significant numerical problems, if $\psi$ and with it $\boldsymbol{H}$ is not computed accurate enough. In that cases a difference field concept is useful to increase the accuracy of the solution [5].

\subsection{Non-linear material properties}

A crucial point for accurate magnetic field computations is the model of the non-linear magnetic material properties of the yoke and of the pole shoes. In general, a vector hysteresis model is necessary to model the magnetization effects within the magnetic domains accurate enough. Here, it is sufficient to apply a scalar model and to approximate the relation between $|\boldsymbol{B}|$ and $|\boldsymbol{H}|$ with a non-linear function (Fig. 1).

Between the measured values, which are drawn in black in Fig. 1, the non-linear function is interpolated by piecewise linear functions. For large values of $|\boldsymbol{H}|$ the non-linear function is linearly extrapolated.

\subsection{Finite element method}

Second order Lagrange elements are used to discretize the computational domain for a solution of the magnetic field problem with the FEM. Applying the Galerkin method yields to

$$
[K(\psi)]\{\psi\}=\{b\}
$$




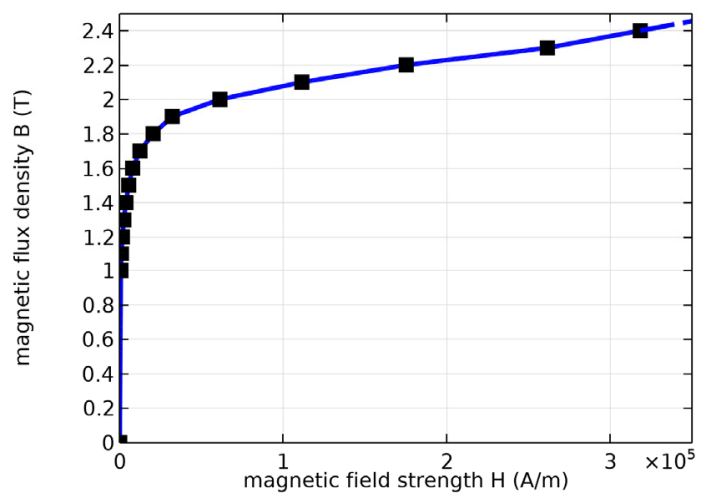

Figure 1: Scalar non-linear model of magnetic material properties.

$[K(\psi)]\{\psi\}=\{b\}$ The stiffness matrix $[K(\psi)]$ depends on the magnetic material properties and therefore on $\psi$. The right-hand side $\{b\}$ contains indirectly the defined magnetization of the permanent magnets. During the derivation of (8) it is assumed that the magnetic flux through the infinite boundary vanishes and that is $\frac{\partial \psi}{\partial n}=0$ respectively.

The non-linear system of equations in (8) is solved using a Newton-Raphson method with re-computation of the tangential matrix within each iteration step. An adaptive control of the damping factor ensures fast convergence.

Within each non-linear iteration step, conjugate gradient (CG) method with algebraic multigrid as preconditioner is used to solve the linear equation system.

After the computation of $\psi, \boldsymbol{B}$ is evaluated in the ROI based on the nodal values of $\psi$ of the finite elements in the ROI. To this end, (1) and (2) are applied with analytical derivatives of the Ansatz functions. However, due to the second order Lagrange polynomials for $\psi$ only first order polynomials for $\boldsymbol{B}$ are obtained. Furthermore, $\boldsymbol{B}$ is discontinuous at element interfaces. There exist several techniques like the patch recovery method to improve the accuracy of $\boldsymbol{B}$ [6]. Another often applied approach is to refine the mesh in the ROI.

\subsection{Boundary element method}

In the case of linear magnetic materials, the BEM is very well suited to solve the Laplace equation for the magnetic scalar potential

$$
\Delta \psi=0
$$

efficiently with high accuracy. Then, only the domain boundaries are discretized with second order Lagrange elements. Applying Galerkin method and Green's theorem

$$
\psi(\boldsymbol{r})=\int_{\partial \Omega} G\left(\boldsymbol{r}, \boldsymbol{r}^{\prime}\right) \frac{\partial \psi\left(\boldsymbol{r}^{\prime}\right)}{\partial n^{\prime}} \mathrm{d} A^{\prime}-\int_{\partial \Omega} \frac{\partial G\left(\boldsymbol{r}, \boldsymbol{r}^{\prime}\right)}{\partial n^{\prime}} \psi\left(\boldsymbol{r}^{\prime}\right) \mathrm{d} A^{\prime}
$$

leads to a linear system of equations

$$
[G]\left\{\frac{\partial \psi}{\partial n}\right\}=[H]\{\psi\}
$$

with dense matrix blocks. The infinite domain boundary is implicitly considered by Green's function of three-dimensional Laplace equation 


$$
G\left(\boldsymbol{r}, \boldsymbol{r}^{\prime}\right)=\frac{1}{4 \pi} \frac{1}{\left|\boldsymbol{r}-\boldsymbol{r}^{\prime}\right|}
$$

Eqn (10) is valid for each computational domain, i.e. for each material domain in practice. Further domains are created by additional cuts of a single domain, which is advantageous for instance in the ROI. The computational domains are coupled applying the boundary conditions (5) and (7). Furthermore, it is not necessary to compute all domains with the BEM, which is the starting point for the proposed coupling with the FEM in the next section.

Considering accuracy of the BEM, it is very important to compute singular and strong singular integrals accurately. Introducing polar coordinates on the boundary elements around the evaluation point, here the integration point of the Galerkin integral, is very advantageous to increase accuracy with acceptable computational costs [7]. These integration techniques are applicable to nearly singular and nearly strong singular integrals, too, which is relevant for accurate field evaluations near domain boundaries, e.g. near the pole shoes in the ROI.

To overcome the large memory requirements and computational costs of the dense matrix blocks in (10), established matrix compression techniques like the adaptive cross approximation (ACA) [8] or the fast multipole method (FMM) [9] are used. The ACA is well suited to solve (10) efficiently, especially in the context of a coupling of the BEM with the FEM [10]. The FMM can be used both for the solution of the equation system and for the post-processing [11]. Furthermore, a modified FMM is the foundation for an efficient meshfree post-processing [12], which is applied here to improve the accuracy in the ROI.

\section{ROBUST AND PRECISE MAGENTIC FIELD COMPUTATION}

A robust and precise method for the computation of magnetic fields is presented here. Besides the solution of the problem, a focus is set on the evaluation of the magnetic fields in the ROI. Starting from the fundamentals of the FEM and the BEM summarized in previous section, an approach is developed, which combines both methods very advantageous for the studied class of field problems. Finally, latest advances in both methods enable a new coupled postprocessing and evaluation of magnetic fields in the ROI.

First, some limits in practical applications of the FEM are shown for precise magnetic field computations. Then, an efficient approach for the solution of the equation system is shown, which prepares an enhanced post-processing, too. This smart coupling of computational methods results then in a powerful and accurate post-processing.

\subsection{Limits of a pure finite element method}

As shown in the previous section, the FEM is in principal very well suited for the type of examined magnetic field problems. All material properties can be considered with desired accuracy and complexity. A refinement of the volume mesh along with a sufficiently large surrounding air domain ensures reliability of the computed fields. However, robustness in the sense of simple to use numerical models is missing. For precise computations, the size of the air domain and the discretization of the ROI must be chosen very carefully.

Here, a three-dimensional model of a magnetic circuit is considered (Fig. 2). Its yoke is made of iron with a non-linear magnetization curve as depicted in Fig. 1. Above and below the air gap for the ROI, permanent magnets with a remanence flux density of $0.6 \mathrm{~T}$ are embedded into the yoke.

Four different numerical cases have been investigated. The radius of the air domain was set to the same value as the size of the yoke and in the second parameter study it was set five times the size of the yoke. Furthermore, two different meshes in the ROI have been created. The magnetic flux density along a line in the ROI for all four cases is shown in Fig. 3. There, 
differences between the four studied cases are visible. That means, the quality of the results strongly depends on the size of the finite elements in the ROI and of the size of the air domain.

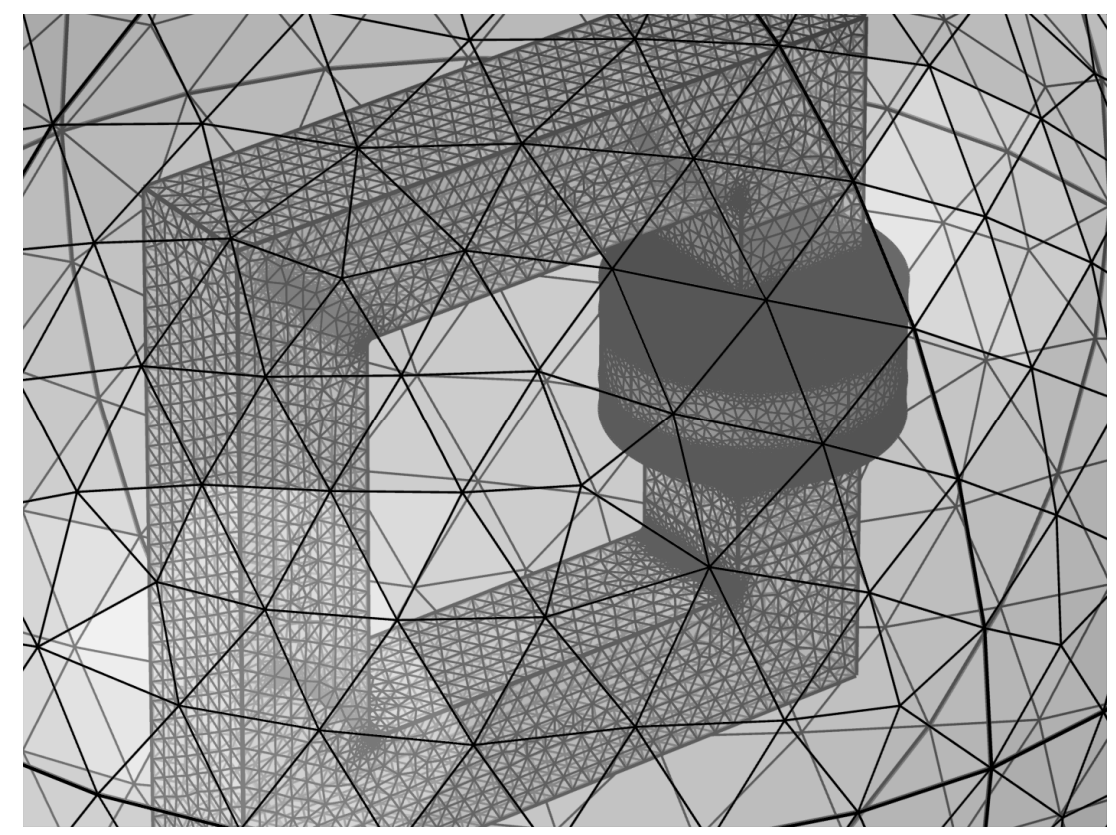

Figure 2: Three-dimensional discretized model of a magnetic circuit.

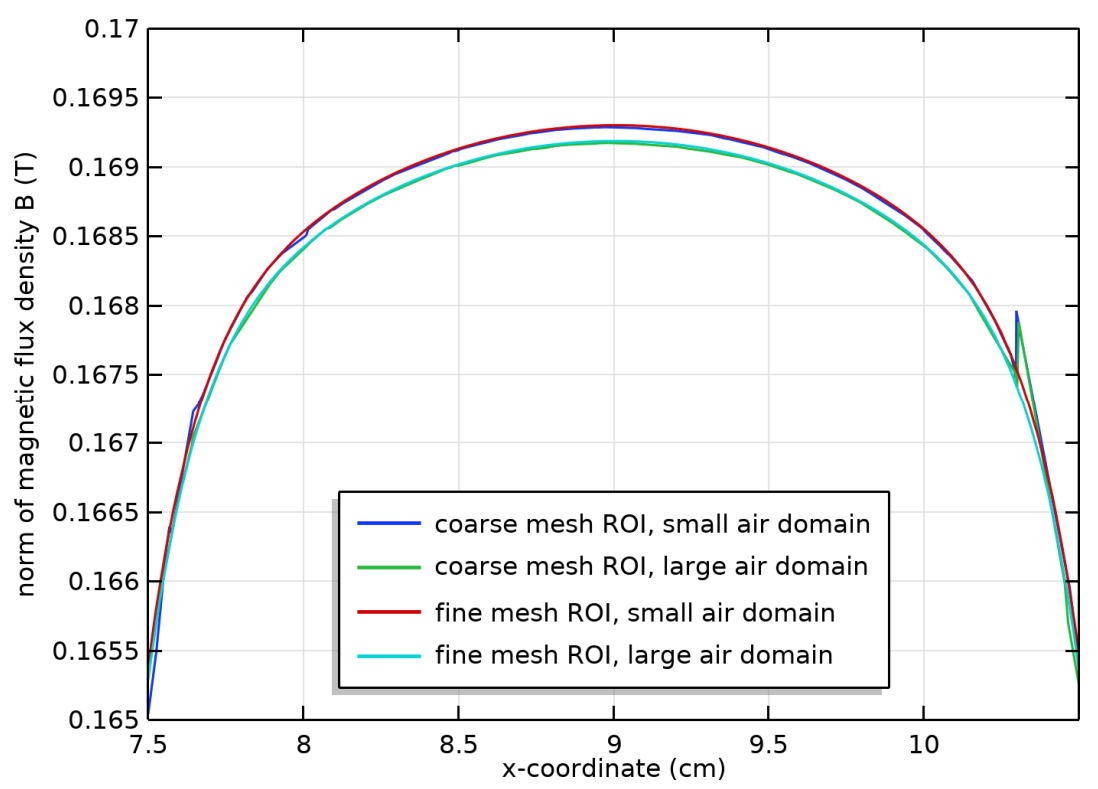

Figure 3: Absolute value of magnetic flux density along a line in the ROI. 


\subsection{BEM-FEM coupling for the solution of the problem}

As demonstrated in the previous sub-section, magnetic field computations in the ROI are sensitive to discretization and size of the air domain. Hence, robustness in an application of the FEM is not guaranteed. To this end, a coupling of the BEM and the FEM is proposed here to improve robustness of magnetic field computations both for the solution of the field problem and for the post-processing.

A coupling of the FEM and the BEM for the solution of magnetic field problems has a long tradition [10]. Latest publications for electromagnetic wave problems [13], acoustics problems [14], or mechanical problems [15] underline that even today a BEM-FEM coupling is competitive and advantageous. However, all recent publications have in common that the post-processing is limited to the computation of field values inside the FEM domain or to only a few characteristic values in the BEM domain.

In the following, the BEM-FEM coupling approach is extended to the post-processing with features comparable to a pure FEM post-processing but with accuracy of BEM. To this end, a propriate pre-processing and solution of the field problem is considered first.

The goal is to compute and to visualize the magnetic field with a high accuracy in the ROI. To overcome interpolation errors caused by the volume discretization of the FEM, the BEM is selected for the ROI. Then, only the artificial boundary of the ROI must be considered to evaluate eqn (10). Normally, the yoke and the pole shoes are discretized using relatively small finite elements. Since the test samples in NMR or ESR applications are positioned in the centre of the ROI, the BEM boundary of the ROI is not placed directly on the surface of the pole shoes but with a small gap to magnetic materials. This enables a layer of finite elements, which increase the element size and hence reduce the number of elements at the BEM domain surface. Then, an efficient solution of the BEM domain based on compression techniques like the ACA or the FMM is possible. A similar approach is used for the air domain. There, also a small FEM mesh reduces the number of boundary elements. In practice, a compromise between computational costs for the BEM with reduced number of elements and for larger FEM domains must be found. Depending on the geometrical configuration of the problem, further parts of the air domain can be solved using the BEM. The proposed splitting is summarized in Fig. 4.

Another advantage of this computational domain splitting is that field singularities, as they occur near edges or corners of the pole shoes, are computed within the FEM domain. There, increasing the number of elements and of integration points is more efficient than in the BEM domain. As a result, relatively smooth boundary values at the BEM domain surfaces are obtained, which can be handled with the techniques presented in [7].

\subsection{BEM-FEM coupling for post-processing}

For a successful magnet design, a powerful post-processing is indispensable. This includes evaluation of fields in defined points, for instance along a line as shown in Fig. 3, visualization of fields in a plane using colours or arrows, or computation and visualization of field lines or isosurfaces. Both accurate values in form of tables or line plots and a threedimensional overview of the field characteristics are required for an engineer.

The challenge of a BEM-FEM post-processing is to combine the two completely different working methods in such a way that this coupling and the splitting of the computational domains is invisible for the magnet designer. A classical approach is, to create in the BEM domains finite elements and to compute the field values in the nodes of these elements [16]. 


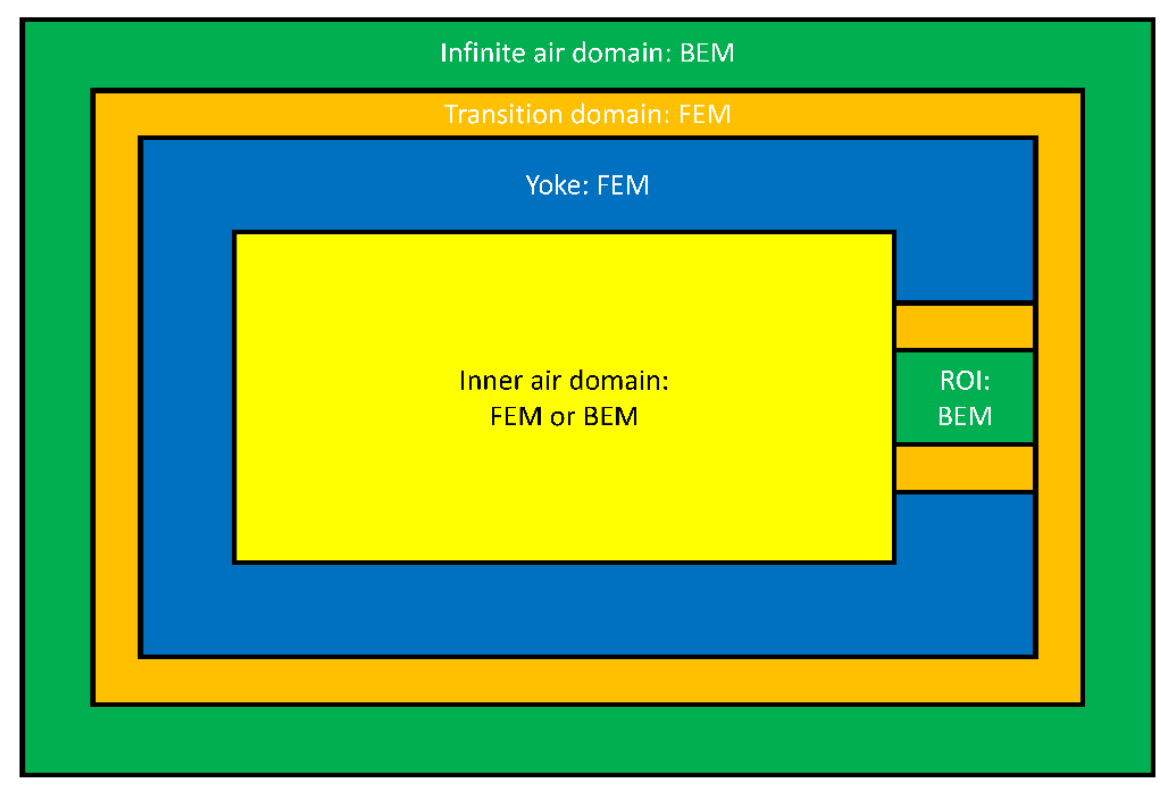

Figure 4: Splitting of computational domains for the BEM or the FEM.

Then, the field values of the FEM and of the BEM are interpolated by standard finite elements in the complete domain and enhanced visualization techniques are applicable [17]. However, that approach requires a large amount of evaluations of eqn (10) along with large computational costs. Hence, an approach, which computes only necessary values, is preferred.

Here, a meshfree post-processing [12] for the BEM is coupled with a classical FEM postprocessing. This requires several steps to split and combine the numerical results. First, the designer defines the type of field evaluation. Then, the post-processing software has to select the relevant computational domain automatically. Values and visualization objects in the FEM domain are computed based on grid values and in the BEM domain a meshfree computation is applied to reduce computational costs and to overcome interpolation inaccuracies. Finally, the computed results are composed for virtual reality (VR) or augmented reality (AR) visualization. To this end, a strictly modular field computation software is required.

The software MuPhyN (multiphysics numerics) of the author integrates modules of a fast BEM solver, a meshfree BEM post-processing, and an interface to a FEM solver, here to the commercial implementation COMSOL Multiphysics (Fig. 5).

A key feature of the BEM post-processing module is that the computation of visualization objects like field lines [18] or isosurfaces [19] is strongly coupled with the BEM. This enables efficient and accurate computations. The visualization objects of BEM and FEM are merged in the open source visualization software COVISE (Fig. 6).

To achieve the required accuracy, the BEM must be appropriate parameterized. Since the transition domain between the yoke and the ROI was introduced, no field singularities occur at the BEM domain surface. That means, smooth values of $\psi$ and $\frac{\partial \psi}{\partial n}$ are obtained at the boundary elements. Hence a relatively coarse mesh suffices. On the one hand, a small number 


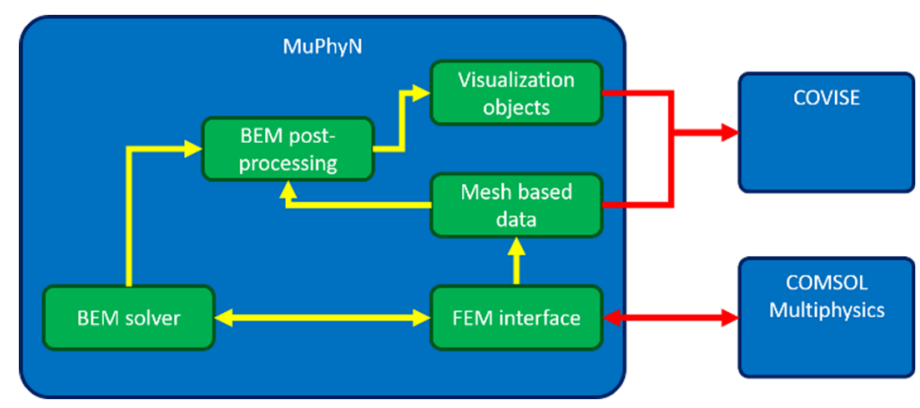

Figure 5: Modular software structure of MuPhyN.

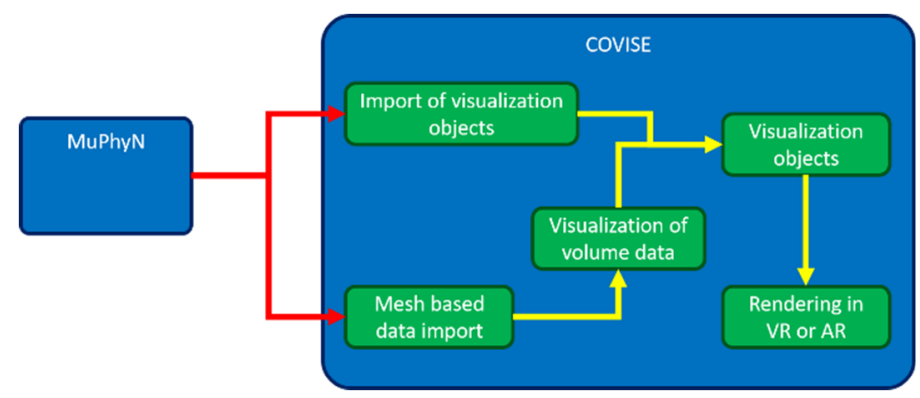

Figure 6: Merging of visualization objects of BEM and FEM.

of boundary elements increases the efficiency of the post-processing in the ROI, on the other hand the relative distance between the evaluation points and the boundary elements can be small. Hence, special treatment of nearly singular and nearly strong singular integrals with sufficiently large number of integration points is necessary [7].

An efficient meshfree BEM post-processing is based on the FMM. The FMM compresses the integrals of eqn (10) and most boundary elements are considered by a series expansion of Green's function in spherical coordinates, e.g. for the single-layer potential part of eqn (10)

$$
\psi(\boldsymbol{r})=\frac{1}{4 \pi} \sum_{n=0}^{L} \sum_{m=-n}^{n} r^{n} Y_{n}^{m}(\theta, \phi) L_{n}^{m} .
$$

The order $L$ adjusts the accuracy of the FMM. The local coefficients $L_{n}^{m}$ contain information of the boundary values of all boundary elements in the far-field of the evaluation point and $Y_{n}^{m}(\theta, \phi)$ are normalized spherical harmonics.

To compute the magnetic field strength $\boldsymbol{H}$ or the magnetic flux density $\boldsymbol{B}$ based on (1), the necessary derivatives can be computed in eqn (13) analytically in Cartesian coordinates. In a similar way, the double-layer part of eqn (10) is considered.

The BEM-FEM post-processing strongly depends on an automatic domain detection method. In the FEM domain, the related finite element of the evaluation point is found with an octree search and an optimization method. The domain detection in the BEM domain is more challenging, since the domain is not discretized [12]. A combination of both approaches determines the relevant domain and computational method automatically and is therefore applicable in algorithms for field line or isosurface computations. 
A bidirectional communication between the software modules in Figs 5 and 6 is necessary to evaluate the magnetic fields across BEM and FEM domain boundaries. In the case of isosurface computations, an exchange of node coordinates improves smoothness of the isosurface, especially if a watertight isosurfaces is determined. For accurate field lines, boundaries must be reliably detected to obtain precise coordinates of the intersection point of the field line with the domain surface. These coordinates are used as start coordinates of the field line segment in the next computational domain.

In total, a BEM-FEM post-processing is based on an automatic domain detection, a modular software concept of numerical methods, and a deep integration of numerical methods with post-processing and visualization techniques. For the latter, access to the implementation of the BEM including compression techniques is necessary to modify these methods with respect to efficiency and accuracy [18], [19]. From point of view of a magnet designer, the proposed approach is easily applicable and transparent concerning the computational method. Depending on the user interface of the field computation software, most steps of the BEM-FEM coupling can be hidden.

\section{NUMERICAL RESULTS}

The proposed BEM-FEM coupling for the computation of magnetic fields has been tested on the magnet introduced in Section 3.1. The yoke and the permanent magnets have been discretized with second order tetrahedral Lagrange elements. A relative fine mesh has been created to consider the non-linear material properties accurately. A relatively coarse mesh for the BEM domain in the ROI and a coarse mesh for the BEM domain of infinite space has been applied (Fig. 7). Between the non-linear FEM domains and the two BEM domains, a FEM domain is added. In total a mesh with 1,232,772 domains and 4,294 boundary elements is obtained.

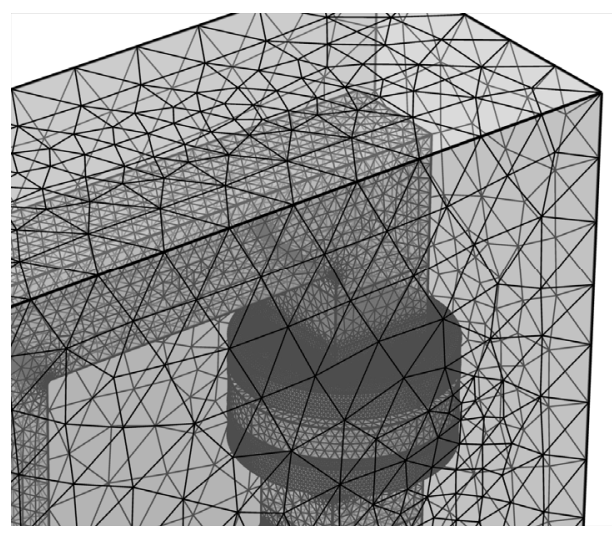

Figure 7: Discretized model of a magnet for a BEM-FEM coupling.

The magnetic properties of the yoke have been modelled based on the non-linear function depicted in Fig. 1. The remanence flux density of the permanent magnets is impressed in the related domains. Since the boundaries of the two BEM domains are in air, continuity of the scalar magnetic potential and of its normal derivative are used as boundary conditions for the BEM-FEM coupling. The ACA has been applied to compress the BEM matrix blocks.

The non-linear problem was solved after three iteration steps of Newton-Raphson method and using BiCGStab solver with direct preconditioner for the underlying linear equation 
system in 55 minutes on an Intel Xeon CPU E3-1275 v5 with $3.6 \mathrm{GHz}$ clock speed. The memory requirements have been 39 GB.

The norm of the magnetic flux density in a plane through the magnet is depicted in Fig. 8. There, the colour plot has been created using a grid both for the FEM and the BEM domain in the shown plane.

Norm of magnetic flux density $B(T)$
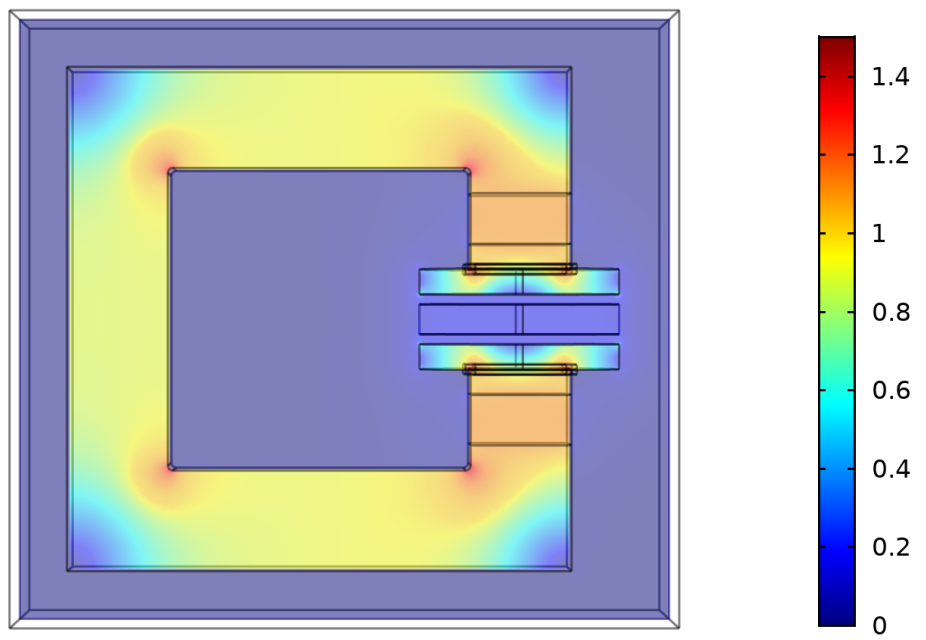

Figure 8: Norm of magnetic flux density computed with BEM and FEM.

The computation of the field lines shown in Fig. 9 has been coordinated by the software MuPhyN. The magnetic flux density in the mesh nodes of the FEM domain was computed in advance and the field lines have been computed using the optimized methods of the FEM solver. In the BEM domain, the field lines haven been obtained using a meshfree BEM postprocessing with FMM compression of integrals.

\section{CONCLUSIONS}

The computation of magnetic fields with a high accuracy, which is required in smart sensor application for NMR or ESR spectroscopy, is implemented by a smart combination and coupling of the finite element method and the boundary element method. The proposed coupling both for the solution of the problem and for the post-processing simplifies the numerical model and ensures reliable numerical results with a high accuracy. In other words, the method is robust and accurate. Thereby, a finite element method in combination with a state-of-the-art boundary element method is very well suited as an easy-to-use and reliable tool for high-end smart sensor design. Mainly the meshfree post-processing of the boundary element method along with an efficient compression technique supports high precision evaluations of magnetic fields in the region of interest. 

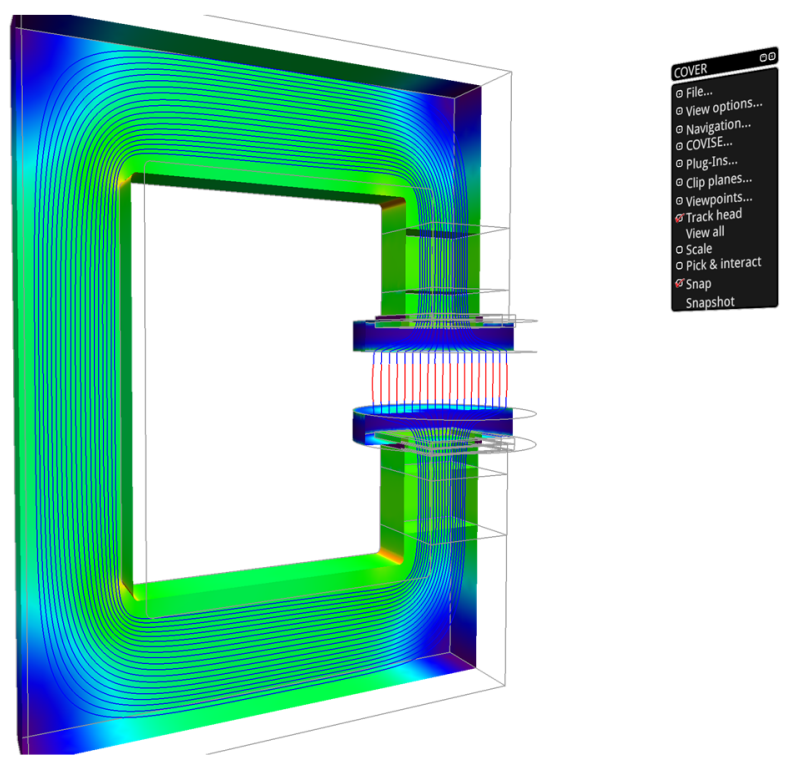

Figure 9: Field lines of the magnetic flux density in the ROI (red) and in the yoke (blue).

\section{REFERENCES}

[1] Meijer, G., Makinwa, K. \& Pertijs, M., Smart Sensor Systems: Emerging Technologies and Applications, Wiley, 2014.

[2] Schlecker, B. et al., VCO-based ESR-on-a-chip as a tool for low-cost, high-sensitivity point-of-care diagnostics. Proceedings of 2017 IEEE SENSORS, Glasgow, pp. 1-3, 2017.

[3] Anders, J., Handwerker, J., Ortmanns, M. \& Boero, G., A low-power high-sensitivity single-chip receiver for NMR microscopy. Journal of Magnetic Resonance, 266, pp. 41-50, 2016.

[4] Alnajjar, B., Buchau, A., Anders, J. \& Blümich, B., An H-shaped low-field permanent magnet for NMR spectroscopy designed using the finite element method. International Journal of Applied Electromagnetics and Mechanics, 61, 2019.

[5] Hafla, W., Buchau, A. \& Rucker, W.M., Accuracy improvement in nonlinear magnetostatic field computations with integral equation method and indirect scalar potential formulations. COMPEL - The International Journal for Computation and Mathematics in Electrical and Electronic Engineering, 25(3), pp. 565-571.

[6] Zienkiewicz, O.C. \& Zhu, J.Z., The superconvergent patch recovery (SPR) and adaptive finite element refinement. Computer Methods in Applied Mechanics and Engineering, 101, pp. 207-224, 1992.

[7] Huber, C.J., Rieger, W., Haas, M. \& Rucker, W.M., The numerical treatment of singular integrals in boundary element calculations. ACES Journal, 12(2), pp. 121126, 1997.

[8] Bebendorf, M., Approximation of boundary element matrices. Numerische Mathematik, 4, pp. 565-589, 2000.

[9] Greengard, L. \& Rokhlin, V., The rapid evaluation of potential fields in three dimensions. Lecture Notes in Mathematics 1360, eds C. Anderson \& C. Greengard, Springer-Verlag: Berlin, pp. 121-141, 1987. 
[10] Buchau, A., Rucker, W.M., Rain, O., Rischmüller, V., Kurz, S. \& Rjasanow, S., Comparison between different approaches for fast and efficient 3D BEM computations. IEEE Transactions on Magnetics, 39(3), pp. 1107-1110, 2003.

[11] Buchau, A., Rieger, W. \& Rucker, W.M., Fast field computations with the fast multipole method. COMPEL - The International Journal for Computation and Mathematics in Electrical and Electronic Engineering, 20(2), pp. 547-561, 2001.

[12] Buchau, A. \& Rucker, W.M., Feasibility of a meshfree post-processing for boundary element methods. WIT Transactions on Modelling and Simulation, vol. 61, WIT Press: Southampton and Boston, pp. 327-338, 2015.

[13] Cvetković, M., Dodig, H. \& Poljak, D., Numerical comparison of compound and extracted eye models for high frequency dosimetry. International Journal for Engineering Modelling, 31(1-2), 2018.

[14] Zhao, W., Zheng, C., Liu, C. \& Chen, H., Minimization of sound radiation in fully coupled structural-acoustic systems using FEM-BEM based topology optimization. Structural and Multidisciplinary Optimization, 58, pp. 115-128, 2018.

[15] Maljaars, P., Kaminski, M. \& den Besten, H., Boundary element modelling aspects for the hydro-elastic analysis of flexible marine propellers. Journal of Marine Science and Engineering, 67(6), 2018.

[16] Hafla, W., Weinläder, A., Bardakcioglu, A., Buchau, A. \& Rucker, W.M., Efficient post-processing with the integral equation method. COMPEL - The International Journal for Computation and Mathematics in Electrical and Electronic Engineering, 26(3), pp. 873-887, 2007.

[17] Buchau, A., Rucker, W.M., Wössner, U. \& Becker, M., Augmented reality in teaching of electrodynamics. COMPEL - The International Journal for Computation and Mathematics in Electrical and Electronic Engineering, 28(4), pp. 948-963, 2009.

[18] Buchau, A. \& Rucker, W.M., Meshfree computation of field lines across multiple domains using fast boundary element methods. IEEE Transactions on Magnetics, 2015.

[19] Buchau, A. \& Rucker, W.M., A meshfree isosurface computation method for boundary element methods. International Journal of Computational Methods and Experimental Measurements, 5(5), pp. 647-658, 2017. 